\title{
COEFICIENTES DE DIGESTIBILIDAD APARENTE DE Thitonia diversifolia Y Cratylia argentea EN CACHAMA BLANCA Y EFECTOS SOBRE LAS VELLOSIDADES INTESTINALES
}

\section{APPARENT DIGESTIBILITY COEFFICIENTS OF Thitonia diversifolia AND Cratylia argentea BY CACHAMA BLANCA AND ITS EFFECTS ON INTESTINAL VILLI}

\author{
Luis Fernando Puerta Rico ${ }^{1}$, José Jaime García González², Jaime Eduardo Parra Suescún², \\ Sandra Clemencia Pardo Carrasco ${ }^{4}$
}

\begin{abstract}
${ }^{1}$ Zootecnista, M.Sc. Circular 2 \# 66b-122, Interior 701, Medellín, Colombia, e-mail: Ifpuertar@unal.edu.co; ${ }^{2}$ Ingeniero Acuícola, M.Sc. Calle 38 sur \# 47a-23, Envigado, e-mail: jjgarciago@unal.edu.co; ${ }^{3}$ Zootecnista, Ph.D. Calle 59 A \# 63-20, Bloque 50 oficina 315, Medellín, Colombia, e-mail: jeparrasu@unal.edu.co; ${ }^{4}$ Médico Veterinario y Zootecnista, Ph.D, Grupo de Investigación BIOGEM, Laboratorio de Modelación Animal -LAMA-, Facultad de Ciencias Agrarias. Universidad Nacional de Colombia, Sede Medellín, Calle 59 A \#63-20, Bloque 50 oficina 309, Medellín, Colombia, e-mail: scpardoc@unal.edu.co
\end{abstract}

Rev. U.D.C.A Act. \& Div. Cient. 20(2): 375-383, Julio-Diciembre 2017

\section{RESUMEN}

En atención a identificar nuevas materias primas fuentes de proteína que no compitan con la alimentación humana, se determinaron los coeficientes de digestibilidad aparente de la proteína (CDA-P), materia seca (CDA-MS) y energía (CDA-E), de botón de oro Tithonia diversifolia y cratylia Cratylia argentea, en Piaractus brachypomus y los efectos sobre las vellosidades intestinales. Para estimar estos coeficientes, se utilizó el método indirecto con marcador, colectando heces. Se utilizaron 120 cachamas (peso promedio $210 \mathrm{~g}$ ), alimentadas con dieta referencia semipurificada (DRS) 69,5\%, 0,5\% de marcador inerte y la materia prima en $30 \%$ de inclusión. Las heces, se recolectaron $10 \mathrm{~h}$ después de haber suministrado el alimento en los tanques, cada hora, durante $12 \mathrm{~h}$. Para medir las vellosidades del intestino anterior, se tomaron muestras en tres tiempos de consumo de las dietas: 0 (Día 0), 5 (Día 5) y 10 días (Día 10). Los CDA-P fueron 69,42\%, para botón de oro y $44,84 \%$, para cratylia, con diferencia estadística. En cuanto a CDA-E, botón de oro fue $67,37 \%$ superior y, estadísticamente, diferente a cratylia $34,27 \%$. Las materias primas causaron denudación en las vellosidades durante los primeros 5 días de consumo; sin embargo, a los 10 días, las vellosidades comenzaron a recuperar su altura, lo que demostró la capacidad de adaptación de la cachama blanca a dietas, con materias primas evaluadas.

Palabras clave: Alimentación y nutrición, materias primas alternativas, Piaractus brachypomus.

\section{SUMMARY}

In order to identify new sources of raw protein materials and not compete with human food, was the apparent digestibility coefficients of protein (CDA-P), dry matter (CDA$\mathrm{MS}$ ) and energy (CDA-E) of mexican sunflower (BO) Tithonia diversifolia and Cratylia (CR) Cratylia argentea, for cachama blanca Piaractus brachypomus and their effects on intestinal villi were determined. To estimate these coefficients, the indirect method with marker, feces collecting were employed. A hundred and twenty white cachamas (average weight $210 \mathrm{~g}$ ), fed on a diet reference semipurified (DRS) $69.5 \%$, $0.5 \%$ inert trace marker ingredient and raw material were evaluated at a level of $30 \%$ inclusion. Feces were collected after 10 hours of food supply each hour during 12 hours. To measure the villi, intestine samples were taken at three times of diet consumption: 0,5 and 10 days. The CDA-P were: $69.42 \%$ for Mexican sunflower and $44.84 \%$ for cratylia, being statistically differet. As for CDA E, Mexican sunflower $67.37 \%$ were higher and statistically different than cratylia $34.27 \%$. Although digestibility values obtained for these raw materials were lower than those reported for raw materials most commonly used in fish feed, the Mexican sunflower approaches to soybean cake. Raw materials caused denudation in the villi during the first few days, however after 10 days the villi started to recuperate its high, showing an adaption of $P$. brachypomus to the evaluated ingredients. 
Key words: Feed and nutrition, Alternative raw materials, Piaractus brachypomus.

\section{INTRODUCCIÓN}

La producción piscícola en Colombia está soportada por tilapias (Oreochromis sp.), trucha arcoiris (Oncorhynchus mykiss) y cachama blanca (Piaractus brachypomus), las cuales, representan el $96 \%$ de la producción nacional total (Cruz-Casallas et al. 2011). De estas tres especies, solo la cachama blanca es originaria del país y se cultiva, gracias a su excelente adaptación a la cría en estanques, su rusticidad, buena conversión alimenticia, resistencia a enfermedades y hábitos alimenticios omnívoros (Vásquez, 2004). El crecimiento de la piscicultura en Colombia ha sido sostenido, pasando de 68.300t, en 2006-2008, a 90.400t, en 2012-2014 (FAO, 2017).

Con el crecimiento de la actividad en el país, el Ministerio de Agricultura y Desarrollo Rural ha generado estrategias para hacerla más competitiva en un mercado globalizado; es por esto que, de acuerdo con MADR \& IICA (2012), a través de la Agenda Nacional de Investigación en Pesca y Acuicultura, se identificaron los principales elementos que requieren atención en la búsqueda de una mayor competitividad. Se reconoce en la agenda, que el insumo más importante es el alimento balanceado, que se encuentra siempre con tendencia al alza de precios y no siempre con las especificaciones técnicas y nutricionales requeridas; por ejemplo, no hay dietas especializadas y para cachama blanca, se utiliza la misma que para tilapias.

Una de las razones del incremento de precio en los alimentos balanceados es la escasez de harina de pescado, como fuente proteica, por consiguiente, la industria acuícola en el mundo está buscando reemplazarla con ingredientes vegetales, como la soya y sus derivados y facilitar así el crecimiento de la industria (Fry et al. 2016). En concordancia con esta situación, una de las demandas tecnológicas definidas en la Agenda Nacional de Investigación en Pesca y Acuicultura (MADR \& IICA, 2012) es adquirir conocimiento acerca de la digestibilidad de materias primas alternativas, para la formulación de nuevas dietas comerciales.

En ese sentido, este estudio buscó probar materias primas no tradicionales en la elaboración de alimentos balanceados para peces y que, adicionalmente, no compitan con la alimentación humana. Asimismo, se buscó identificar el efecto de las materias primas sobre la unidad funcional de absorción del intestino, la vellosidad.

El tracto gastrointestinal es el encargado de procesar y de digerir el alimento para degradarlo en formas que puedan ser absorbidas y asimiladas; por lo tanto, es importante estudiar su histología, con el objetivo de establecer su integridad y entender el efecto de los alimentos sobre su estructura (Sweetman et al. 2008).

Consecuentemente, es correcto pensar que los análisis de digestibilidad de las materias primas deben ser acompañados de estudios de integridad intestinal. Elementos, como la longitud de la vellosidad, pueden ser utilizados como indicador del efecto del alimento sobre la integridad del tracto gastrointestinal.

\section{MATERIALES Y MÉTODOS}

Localización. El experimento, se llevó a cabo en el Laboratorio Experimental de Alimentación y Nutrición de Peces del Instituto de Acuicultura de la Universidad de los Llanos (IALL), ubicado en el kilómetro 4 vía Puerto López, en la vereda Barcelona, de la ciudad de Villavicencio, en el departamento del Meta (Colombia), a $418 \mathrm{msnm}$. La temperatura promedio anual es de $25^{\circ} \mathrm{C}$, la precipitación de $4.050 \mathrm{~mm} /$ año y la humedad relativa de $75 \%$. La región está clasificada como bosque pluvial tropical, según las zonas de vida de Holdridge.

Dietas experimentales. Las dietas utilizadas en este experimento, para determinar el coeficiente de digestibilidad aparente (CDA) de cada materia prima, se elaboraron con base en una dieta referencia semipurificada (DRS) (Tabla 1), propuesta por Vásquez-Torres et al. (2002) y mezclada en una relación de $69,5 \%$, de DRS, 30\%, de una materia prima y $0,5 \%$, de marcador. Las materias primas estudiadas fueron botón de oro Thitonia diversifolia (BO) y cratylia Cratylia argentea (CR). Como marcador inerte, se utilizó óxido de cromo $\left(\mathrm{Cr}_{2} \mathrm{O}_{3}\right)$, como se aprecia en la tabla 1 .

Para proceder a la elaboración de las dietas, se colectaron hojas verdes sin tallos de cada materia prima y la cosecha de las hojas, se hizo teniendo en cuenta el intervalo de corte recomendado, para obtener el mejor contenido nutricional. La cratylia fue cortada a los 60 días del último corte (Rincón et al. 2007) y el botón de oro, a los 50 (Mahecha \& Rosales, 2005). Las materias primas, se sometieron a un proceso de secado natural al sol, se molieron a un tamaño de partícula entre 0,3 y 0,4mm y se enviaron al Laboratorio de Análisis Químico y Bromatológico de la Universidad Nacional de Colombia, Sede Medellín, para realizar el análisis proximal (materia seca, proteína bruta, extracto etéreo, fibra bruta, ceniza e energía bruta) (AOAC, 2012), que se presenta en la tabla 2.

Las dietas experimentales pasaron por un proceso de extrusión, secadas al horno por $12 \mathrm{~h}$, a $60^{\circ} \mathrm{C}$ y luego, almacenadas a $4^{\circ} \mathrm{C}$, hasta su distribución. Las muestras que se tomaron de las materias primas, dieta referencia y dietas 
Tabla 1. Composición de la dieta referencia semipurificada (DRS) ${ }^{1}$ utilizada en la determinación de coeficientes de digestibilidad aparente, en cachama blanca Piaractus brachypomus ${ }^{2}$.

\begin{tabular}{|l|c|}
\hline \multicolumn{1}{|c|}{ Ingredientes } & $\mathbf{g} / \mathbf{1 0 0} \mathbf{g}$ dieta \\
\hline Caseína & 33,3 \\
\hline Gelatina & 3,4 \\
\hline Dextrina & 40 \\
\hline Alfa-celulosa & 14,1 \\
\hline Aceite de pescado & 2,4 \\
\hline Aceite vegetal & 2,4 \\
\hline Premezcla vitaminas & 0,2 \\
\hline Premezcla microminerales & 0,1 \\
\hline Premezcla macrominerales & 4 \\
\hline Vitamina C & 0,1 \\
\hline
\end{tabular}

${ }^{1}$ Composición analizada de la DRS: MS 93\%; PB 86,42\%; lípidos 2,29\%, cenizas 3,66\% ${ }^{2}$ Composición analizada: MS 91\%; PB 94,02\%. ${ }^{3}$ Rovimix Vitaminas Lab. Roche S.A: Vit A 8.0*106UI, Vit D3, 1.8*106UI, Vit E 66,66g, Vit B1 6,66 g, Vit B2 13,33g, Vit B6 6,66g, Pantotenato de Ca 33,33g, Biotina 533,3mg, Ac. Fólico 2,66g, Ac. Ascórbico 400g, Ac. Nicotínico 100g, Vit B12 20mg, Vit K3 6,66g, vehículo csp 1,0kg. ${ }^{4}$ Premix microminerales Lab. Roche S.A.: Composición por 100g: Mg1,0, Zn 16,0, Fe 4,0, Cu 1,0, I 0,5, Se 0,05. Co 0,01. ${ }^{5}$ Composición por $100 \mathrm{~g}$ de mezcla: $\mathrm{Ca}\left(\mathrm{H}_{2} \mathrm{PO}_{4}\right)$ 13,6g; Lactato de Ca 34,85g; $2 \mathrm{MgSO}_{4} .7 \mathrm{H}_{2} \mathrm{O}, 13,2 \mathrm{~g} ; \mathrm{KH}_{2} \mathrm{PO}_{4}$ 24g; $\mathrm{NaCl} 4,5 \mathrm{~g} ; \mathrm{AlCl}_{3}$ 0,015g, CMC 9,835g. ${ }^{6}$ Rovimix Stay-C35, DSM. ${ }^{2}$ Vásquez et al. (2002).

Tabla 2. Análisis proximal de harinas de hojas de botón de oro Thitonia diversifolia y cratylia Cratylia argentea.

\begin{tabular}{|c|c|c|c|c|c|c|c|}
\hline Materia prima/ nutrientes & MS\% & $\begin{array}{c}\text { PB } \\
\%\end{array}$ & EE\% & FDN \% & $\begin{array}{c}\text { FDA } \\
\%\end{array}$ & $\begin{array}{c}\text { CZ } \\
\%\end{array}$ & $\begin{array}{c}\text { EB Kcal/kg } \\
\text { MS }\end{array}$ \\
\hline $\begin{array}{c}\text { Botón de oro (BO) } \\
\text { (50 días de corte) }\end{array}$ & 96,6 & 23,1 & 2,2 & 27,7 & 25,3 & 16,9 & 3907,2 \\
\hline $\begin{array}{c}\text { Cratylia (CR) } \\
\text { (60 días de corte) }\end{array}$ & 99 & 16,5 & 2,1 & 46,4 & 38,1 & 5,8 & 4692,2 \\
\hline
\end{tabular}

${ }^{1}$ PB \% Porcentaje de proteína bruta, EE\% Porcentaje de extracto etéreo, FDA\% Fibra en detergente ácido, FDN\% Fibra en detergente neutro tratado con amilasa, CZ\% porcentaje de cenizas, EB Kcal/kilo MS Energía bruta en kilocalorías por kilo de materia seca.

experimentales, fueron almacenadas en bolsas selladas, hasta el momento de su análisis proximal (Tabla 3).

Se utilizaron 120 juveniles de cachama blanca, con un peso promedio inicial de $210 \pm 10 \mathrm{~g}$, obtenidos de los estanques de levante del Instituto de Acuicultura de la Universidad de los Llanos, lugar en el que se suministró un alimento comercial para peces, del $32 \%$ de proteína bruta. Las cachamas, se distribuyeron en dos lotes homogéneos de 60 unidades y se depositaron en dos piletas circulares, cada una con capacidad de 3.500L, las cuales, a su vez, sirvieron como sitio de adaptación para los peces. Mientras los peces permanecieron en estas piletas, se alimentaron con la dieta referencia semipurificada (DRS) durante cinco días; a partir del sexto día, a cada pileta se asignó una de las dietas experimentales: DBO (botón de oro) y DCR (cratylia) y se abasteció a los peces, de cada pileta, la dieta asignada, hasta saciedad aparente, dos veces al día (9:00 y 15:00 horas), durante 15 días. Luego de la última alimentación en el día 15, de cada tratamiento, se seleccionaron 21 peces, que fueron distribuidos en tres tanques cónicos de 200L, para determinar la digestibilidad, con sistema Guelph modificado. Los tanques cónicos, en los que fueron depositados los peces, contaban con un sistema de recirculación, compuesto por dos filtros mecánicos y 
Tabla 3. Composición proximal de la dieta referencia semipurificada y de las dietas experimentales con botón de oro Thytonia diversifolia y cratylia Cratylia argentea, en cachama blanca Piaractus brachypomus.

\begin{tabular}{|c|c|c|c|c|c|}
\hline Dietas/ $^{\text {nutrientes }}{ }^{\mathbf{1}}$ & MS\% & PB\% & EE\% & CZ\% & EB Kcal/kg \\
\hline Referencia (DRS) & 95,4 & 32,8 & 7,9 & 4,0 & 4632,9 \\
\hline Botón de oro (DBO) & 97,8 & 29,2 & 8,2 & 7,8 & 4492,2 \\
\hline Cratylia (DCR) & 98,4 & 27,4 & 8,6 & 4,6 & 5008,3 \\
\hline
\end{tabular}

${ }^{1} \mathrm{~PB} \%$ Porcentaje de proteína bruta, EE\% Porcentaje de extracto etéreo, CZ\% porcentaje de cenizas, EB Kcal/kg MS Energía bruta en kilocalorías por kg de materia seca.

cuatro biofiltros ubicados en serie, lo que permitió mantener un flujo constante de $1 \mathrm{~L} / \mathrm{min} /$ tanque y una calidad del agua, apropiada para la especie. En estos tanques cónicos no se suministró alimento, los peces permanecieron allí por 22 horas, solamente para recolección de heces, destinadas al análisis de la digestibilidad de las dietas. El oxígeno disuelto, se mantuvo en $7,9 \mathrm{mg} / \mathrm{L}$, gracias a una permanente aireación. Otras características del agua fueron temperatura $25^{\circ} \mathrm{C} ; \mathrm{pH}$ 7,1; dureza de $40 \mathrm{mg} / \mathrm{L}$; alcalinidad de $70 \mathrm{mg} / \mathrm{L}$ y nitritos, nitratos y amonio, por debajo de $0,02 \mathrm{mg} / \mathrm{L}$.

Luego de $10 \mathrm{~h}$ de permanecer los peces en los tanques cónicos, tiempo en el cual el alimento ha recorrido el tracto gastrointestinal, de acuerdo con Vásquez-Torres et al. (2002), se comenzó a recolectar heces, con intervalos de 1h, durante 12h. En cada recolección, se retiró el exceso de agua de las heces y de inmediato se llevaron a estufa, a una temperatura de $60^{\circ} \mathrm{C}$, por $24 \mathrm{~h}$. Una vez las muestras estuvieron secas, se pulverizaron y se congelaron para, posteriormente, realizar los análisis de laboratorio.

La determinación de la proteína, se llevó a cabo con el método de Kjeldah (1883). El valor energético de las materias primas fue medido en una bomba calorimétrica (AOAC, 2012) y para medir la concentración de óxido de cromo, se utilizó el método de digestión ácida, propuesto por Furukawa \& Tsukahara (1966).

Para la determinación de los coeficientes de digestibilidad aparente, se aplicó el método indirecto, propuesto por Cho et al. (1985). En la determinación del coeficiente de digestibilidad aparente de la proteína y de la energía de todas las dietas se calcularon utilizando la ecuación de Nose (1966):

CDANut(\%) $=100-\left[100 x\left(\% \mathrm{Cr}_{2} \mathrm{O}_{3} \mathrm{~h} / \% \mathrm{Cr}_{2} \mathrm{O}_{3} \mathrm{~d}\right) \times(\%\right.$ Nuth/\%Nutd $\left.)\right]$ Donde:

CDANut $=$ Coeficiente de digestibilidad aparente del nutriente

$\% \mathrm{Cr}_{2} \mathrm{O}_{3} \mathrm{~d}=$ Porcentaje de óxido de cromo de la dieta.

$\% \mathrm{Cr}_{2} \mathrm{O}_{3} \mathrm{~h}=$ Porcentaje de óxido de cromo en las heces
$\%$ Nutd $=$ Porcentaje de nutriente en la dieta

$\%$ Nuth $=$ Porcentaje de nutriente en las heces

La digestibilidad total de cada materia prima, se estableció por el método de la diferencia, teniendo en cuenta la ecuación descrita por Bureau \& Hua (2006):

CDAmp $=$ CDANutde $+[$ CDANutde - CDANutds $*(X *$ Dds $/$ Y*Ding)]

Donde:

CDAmp $=$ Coeficiente de digestibilidad de la materia prima CDANutde $=$ Coeficiente de digestibilidad aparente del nutriente dentro de la dieta experimental

CDANutds $=$ Coeficiente de digestibilidad aparente dentro de la dieta semipurificada

Dds $=$ Porcentaje del ingrediente dentro de la dieta semipurificada

Ding $=$ Porcentaje del nutriente en la materia prima evaluada

$X=$ Proporción de la dieta semi purificada (69.5\%);

$\mathrm{Y}=$ Proporción de la materia prima (30\%).

Análisis morfométrico intestinal. Para determinar el efecto del consumo de las dietas sobre la morfología de las vellosidades intestinales, se realizó eutanasia humanitaria a seis peces de cada tratamiento, durante los tres días de medición, definidos por los días que llevaban consumiendo la dieta:

Día 0. Tiempo inicial, en el cual, se distribuyeron los peces en las dos piletas. Estos seis peces representaron el grupo de referencia para verificar el estado general de salud y la evaluación macroscópica del estado de los órganos de los animales, antes de suministrar las dietas experimentales. Día 5 y 10 . Después de cinco y diez días de estar consumiendo las dietas con las materias primas, respectivamente.

Para la obtención de las diferentes muestras de intestino, los peces fueron previamente anestesiados con aceite de clavo, a razón de $60 \mathrm{mg} / \mathrm{L}$, hasta que se observó una pérdida horizontal del eje de nado; se pesaron, se midieron y se procedió a la obtención del intestino. El abordaje a la 
cavidad celómica, se realizó mediante un corte parabólico ascendente para exponer el tracto gastrointestinal. Se retiró todo el paquete de vísceras y se tomó cuidadosamente el tracto gastrointestinal por completo; se separó el intestino y se inyectó formaldehido buferado al $4 \%$, para fijación. Se efectuó una inmersión en bloques de parafina y cortes de $3 \mu \mathrm{m}$ en micrótomo; a los cortes, se les realizó tinción con hematoxilina-eosina (H-E). Posteriormente, se realizó $\square$ la toma de material fotográfico del intestino anterior, con microscopio 10x9 de luz Nikon Eclipse E600; cámara Nikon Cam DMX 1200 y el uso del programa de captura y manejo de imágenes (ACT-1®). La longitud de las vellosidades intestinales, se midió desde la base de la vellosidad hasta el ápice, que se encuentra hacia el lumen del órgano, tomando, en promedio, diez vellosidades por campo de microscopio.

Diseño y análisis estadístico. Para el caso del análisis de los coeficientes de digestibilidad de las materias primas, se utilizó un modelo lineal general (GLM) completamente al azar, en donde solo había un factor a probar. Se eligió un nivel de significancia del $5 \%$; en los casos en que se hallaron diferencias estadísticas significativas, se llevaron a cabo las correspondientes pruebas de Tukey. Para el análisis del efecto sobre la altura de las vellosidades intestinales, se consideró una estructura factorial (Steel \& Torrie, 1997), donde los factores en cuestión fueron materia prima (botón de oro y cratylia) y tiempo de consumo de la dieta con la materia prima (días 0,5 y 10); cada tratamiento tuvo tres repeticiones. Se usó el software SAS ${ }^{\circledR} 9.1$ y se verificó el cumplimiento del supuesto de normalidad de los errores.

\section{RESULTADOS Y DISCUSIÓN}

Los peces que consumieron las dietas experimentales no presentaron signo alguno de enfermedad que causara su retiro o sacrificio inmediato. Además, el suministro de las dietas a saciedad aparente, no generó sobrantes.
El coeficiente de digestibilidad aparente (CDA) es una forma de medir indirectamente la absorción de un nutriente, debido a que no es suficiente que los nutrientes se encuentren en altos porcentajes, sino que, además, deben ser digeribles y aprovechados por el pez (Manríquez, 1994). Los diferentes métodos usados para su determinación (disección, extrusión, Guelph \& Guelph modificado) pueden ser usados, siempre y cuando, se apliquen con rigurosidad (Abimorad \& Carneiro, 2004).

A mayor CDA mejor será el aprovechamiento del nutriente de la materia prima por parte del organismo en cuestión. Los CDA para materia seca (CDA-MS), proteína bruta (CDA-PB) y energía digestible (CDA-ED) para cada materia prima, se presentan en la tabla 4.

Los CDA del botón de oro en cachama blanca permiten comparar esta materia prima con otras de uso tradicional. Cuando se compara con los CDA para maíz, soya y trigo, reportados por Vásquez et al. (2013), para la misma especie, se observa en el botón de oro mayor CDA que el maíz y alrededor de los del trigo y soya (Tabla 5). Cuando se compara la digestibilidad del botón de oro con la harina de pescado, esta última es mejor, lo que podría sugerir que el botón de oro, como fuente proteica, estaría más cerca de la soya que de la harina de pescado y pudiese ser considerada como una fuente alternativa. En general, los CDA para botón de oro del presente estudio difieren de los reportados por Ortiz-González et al. (2014) (Tabla 5). Aunque, los análisis de digestibilidad fueron realizados en el IALL, bajo la misma metodología y usando los mismos equipos, tanto humanos como técnicos, las diferencias pueden estar asociadas a la calidad de la materia prima; Ortiz-González et al. (2014) no mencionan la edad de corte de las hojas, ni reportan los porcentajes y los componentes de la fibra en los análisis proximales de las materias primas. Para el caso específico de botón de oro, en el presente estudio, la cantidad de fibra en detergente neutro

Tabla 4. Coeficientes de digestibilidad de materia seca, proteína y energía para el botón de oro Thytonia diversifolia y cratylia Cratylia argentea, en cachama blanca Piaractus brachypomus.

\begin{tabular}{|c|c|c|c|c|c|}
\hline $\begin{array}{c}\text { Dietas/ Coeficiente } \\
\text { de digestibilidad } \\
\text { aparente (CDA) }\end{array}$ & $\begin{array}{c}\text { CDA-Materia } \\
\text { Seca (\%) }\end{array}$ & $\begin{array}{c}\text { CDA-Proteína } \\
\text { Bruta (\%) }\end{array}$ & $\begin{array}{c}\text { Proteína } \\
\text { Digestível (\%) }\end{array}$ & CDA-ED (\%) & $\begin{array}{c}\text { Energía } \\
\text { Digestible } \\
\text { (kcal/kg) }\end{array}$ \\
\hline Referencia (DRS) & 79,92 & 95,37 & --- & 81,47 & --- \\
\hline Botón de oro (DBO) & $67,37^{\mathrm{a}}$ & $69,42^{\mathrm{a}}$ & 16 & $67,37^{\mathrm{a}}$ & 2632,4 \\
\hline Cratylia (DCR) & $16,03^{\mathrm{b}}$ & $44,84^{\mathrm{b}}$ & 7,4 & $34,26^{\mathrm{b}}$ & 1608 \\
\hline EEM & 1,31 & 1,74 & ---- & 1,01 & -- \\
\hline
\end{tabular}

${ }^{a}$ Promedios \pm DS con diferente letra en la misma columna son diferentes estadísticamente por prueba de Tukey $(\mathrm{P}<0,001)$. EEM: Error estándar de la media. 
(FDN) y de fibra en detergente ácido (FDA) (Tabla 2) indican niveles bajo-medios de fibra, en comparación con los niveles de Cratylia, representados, principalmente, por menores niveles de celulosa y de lignina (FDA). Esta condición, de bajo contenido de fibra en la materia prima, pudo ser la responsable de una mayor digestibilidad.
Por otro lado, la cratylia presentó bajos CDA y la cantidad de fibra fue muy alta: FDN y FDA (Tabla 2), lo cual, podría explicar la baja digestibilidad. Adicionalmente, en el presente estudio, se consideró un tiempo de adaptación a las dietas con la materia prima de 15 días, mientras que en el estudio de Ortiz-González et al. (2014) fue de 7 días, disminuyendo

Tabla 5. Comparación de los coeficientes de digestibilidad aparente de materia seca (CDA-MS), proteína bruta (CDAPB) y energía digestible (CDA-ED), de materias primas tradicionales y no tradicionales, para cachama blanca Piaractus brachypomus, obtenidos por varios investigadores.

\begin{tabular}{|l|c|c|c|c|c|c|}
\hline $\begin{array}{c}\text { Materias primas } \\
\text { CDA (\%) }\end{array}$ & Maíz americano & $\begin{array}{c}\text { Torta de } \\
\text { soya }\end{array}$ & $\begin{array}{c}\text { Harina de } \\
\text { Trigo duro }\end{array}$ & $\begin{array}{c}\text { Harina de } \\
\text { pescado }\end{array}$ & Botón de oro & Cratylia \\
\hline CDA-MS & $59,9^{\mathrm{a}}$ & $62,5^{\mathrm{a}}$ & $53,5^{\mathrm{a}}$ & $76,2^{\mathrm{a}}$ & $35,57^{\mathrm{b}}$ & $5,42^{\mathrm{b}}$ \\
\hline CDA-PB & $69,0^{\mathrm{a}}$ & $92,1^{\mathrm{a}}$ & $86,4^{\mathrm{a}}$ & $85,0^{\mathrm{a}}$ & $35,73^{\mathrm{b}}$ & $29,71^{\mathrm{b}}$ \\
\hline CDA-ED & $57,6^{\mathrm{a}}$ & $79,3^{\mathrm{a}}$ & $67,1^{\mathrm{a}}$ & $84,0^{\mathrm{a}}$ & $37,56^{\mathrm{b}}$ & $12,66^{\mathrm{b}}$ \\
\hline CDA-MS & & & & & $67,37^{\mathrm{c}}$ & $16,03^{\mathrm{c}}$ \\
\hline CDA-PB & & & & & $69,42^{\mathrm{c}}$ & $44,84^{\mathrm{c}}$ \\
\hline CDA-ED & & & & & $67,37^{\mathrm{c}}$ & $34,26^{\mathrm{c}}$ \\
\hline
\end{tabular}

a'Vásquez et al. (2013), ' Ortiz-González et al. (2014), ' Presente estudio.

el efecto abrasivo de este componente antinutricional. Estas diferencias en CDA, con la misma materia prima, permiten sugerir que la metodología de análisis debe incluir una completa descripción del estado de corte de la planta, así como un análisis proximal, que contemple la descomposición y la determinación de los componentes fibrosos. De otra parte, los tiempos de consumo de la dieta, se debieran considerar, por lo que eventos fisiológicos de adaptación a las materias primas podrían suceder y esto mejoraría la digestibilidad.

En un cultivo experimental de cachama blanca (peso inicial de 267g, 59 días de cultivo, densidad de 0,8 peces $/ \mathrm{m}^{2}$ ), Contreras Castro \& Canchila Asencio (2012) encontraron ganancias de peso y conversiones alimenticias estadísticamente diferentes, cuando compararon un alimento balanceado comercial, del $24 \%$ de proteína bruta (6,08/día y conversión de 1,14), frente a ese mismo, con un reemplazo del $15 \%$, con botón de oro (4,85g/día y conversión de 1,58$)$. Esos resultados permiten presumir que, a pesar de los CDA encontrados en el presente estudio, el botón de oro requiere mayores estudios antes de recomendar su uso en la dieta de cachama blanca. El presente estudio no tuvo etapa de cultivo con dietas convencionales e inclusión de las materias primas, apenas se llegó hasta la determinación de los CDA, con dietas de referencia semipurificadas; en consecuencia, es necesario una posterior investigación, para observar dichos efectos productivos.
En cuanto a la altura de las vellosidades del intestino anterior, no se observaron interacciones entre las materias primas y los días de consumo; entonces, se analizaron los efectos principales por separado y se encontró un efecto significativo estadísticamente, del factor tiempo $(P=0,008)$, sobre la altura de las vellosidades (Tabla 6). Las vellosidades del intestino anterior, luego de cinco días de consumo de las dietas con las materias primas, sufrieron un proceso de denudación y para el día 10, aumentaron nuevamente de tamaño; la recuperación es total con botón de oro, mientras que con cratylia se recupera, pero no alcanza la longitud inicial (Tabla 6). El cambio en el tamaño de las vellosidades a los cinco y diez días de consumo podría estar relacionado al proceso de adaptación del intestino a las materias primas, de acuerdo a lo reportado por Franco \& Quintero (2013); sin embargo, no se encontraron estudios en cachama blanca que hayan sido alimentadas con botón de oro o cratylia y determinado, simultáneamente, el desarrollo de las vellosidades intestinales. Franco \& Beltrán (2012) alimentaron cachamas blancas con una dieta que tenía $15 \%$ de morera Morus alba, otra planta usada tradicionalmente en la producción del gusano de seda y ahora en la alimentación animal y registraron cambios morfométricos en el intestino anterior. Los peces alimentados con morera presentaron vellosidades con $533,1 \pm 126,9 \mu \mathrm{m}$, mientras que aquellos que no consumieron la planta, las tuvieron de $602,6 \pm 128,7 \mu \mathrm{m}(\mathrm{P}<0,05)$. Posteriormente, Franco \& Quintero (2013) procedieron con otro experimento, donde 
aumentaron a $20 \%$ la inclusión de morera y lo compararon con una dieta testigo. En este caso, las vellosidades del intestino anterior fueron de $765 \pm 156 \mu \mathrm{m}$, en los peces que consumieron morera, mientras que para los que consumieron el alimento balanceado fueron de $952 \pm 139 \mu \mathrm{m}(\mathrm{P}<0,05)$. Lo anterior denota un efecto abrasivo de los componentes antinutricionales de la morera sobre la vellosidad. De la misma forma, para el caso de botón de oro y cratylia, en el presente estudio, se observa una pérdida de altura de la vellosidad. De hecho, los resultados obtenidos por Franco \& Quintero (2013) muestran que el uso de materias primas alternativas, con una cantidad apreciable de fibra, generan cambios en la morfología del intestino, con efectos sobre la velocidad de tránsito del bolo alimenticio por el tracto gastrointestinal, con efectos, tanto en la absorción de nutrientes como en la morfología del intestino.
De acuerdo con Sweetman et al. (2008), una gran superficie en el tracto gastrointestinal es necesaria para una óptima absorción de nutrientes y es una importante barrera contra patógenos y si esta capa de células epiteliales está afectada, se alteran sus funciones digestivas e inmunológicas. Si se busca la relación entre los coeficientes de digestibilidad aparente de las materias primas estudiadas y sus efectos sobre la vellosidad intestinal, se puede decir que, independiente de la materia prima, hay un efecto sobre la integridad de la vellosidad.

Para el caso del botón de oro, con CDA superiores y diferentes estadísticamente a los de cratylia, se observó una recuperación de la vellosidad al día 10 de consumo, no así para la cratylia, lo que pudiese ser correlacionado con la baja digestibilidad y los altos contenidos de fibra de la cratylia.

Tabla 6. Efecto de la inclusión de botón de oro Thitonia diversifolia y cratylia Cratylia argentea en la dieta de referencia semipurificada y los días de consumo de la dieta, sobre la altura (ųm) de las vellosidades del intestino anterior, en cachama blanca Piaractus brachypomus.

\begin{tabular}{|l|c|c|c|c|}
\hline \multicolumn{1}{|c|}{ Dieta } & Tiempo 0 & Tiempo 1 & Tiempo 2 & EEM \\
\hline Botón de oro & $637,2^{\text {ax }}$ & $373,7^{\text {bx }}$ & $674,3^{\text {acx }}$ & \multirow{2}{*}{11,30} \\
\hline Cratylia & $637,2^{\text {ax }}$ & $497,5^{\text {by }}$ & $559,2^{\text {cy }}$ & \\
\hline
\end{tabular}

${ }_{a, b, c}$ Dentro de una misma fila, medias con diferente letra son estadísticamente diferentes $(P<0,001){ }^{x, y}$ Dentro de una misma columna, medias con la misma letra no difieren estadísticamente $(P<0,001)$. EEM: Error estándar de la media.

De acuerdo con Fabregat et al. (2011), algunos ingredientes fibrosos pueden afectar negativamente las características del epitelio intestinal de juveniles de Piaractus mesopotamicus; sin embargo, se sugiere que para establecer la relación entre CDA y arquitectura intestinal, se realicen estudios sobre las células que conforman las vellosidades y mediciones de las criptas, en las mismas.

Los resultados nos permiten concluir que el botón de oro, con una adecuada edad de corte, puede llegar a ser incluido en las dietas para cachama blanca, debido a sus altos valores de CDA. La cachama blanca es un pez con una gran capacidad de adaptarse a numerosas ofertas alimenticias en el medio ambiente, gracias a la capacidad de los peces de modular y adaptar su tracto gastrointestinal a las condiciones a las que sean expuestas. Este trabajo permite recomendar que los estudios de digestibilidad sean acompañados con estudios de integridad intestinal.

Agradecimientos y financiación. Los autores agradecen a la Universidad Nacional de Colombia, por la financiación al proyecto código 21485. Conflictos de intereses: El manuscrito fue preparado y revisado con la participación de todos los autores, quienes declaramos que no existe conflicto de intereses que ponga en riesgo la validez de los resultados presentados. El estudio contó con el Aval del Comité de Ética de la Universidad Nacional de Colombia Sede Medellín CEMED-018, del 10 de marzo del 2014.

\section{BIBLIOGRAFÍA}

1. ABIMORAD, E.G.; CARNEIRO, D.J. 2004. Métodos de Coleta de Fezes e Determinação dos Coeficientes de Digestibilidade da Fração Protéica e da Energia de Alimentos para o Pacu, Piaractus mesopotamicus (Holmberg, 1887). R. Bras. Zootec. 33 (5):1101-1109.

2. AOAC. 2012. Official methods of analysis. Association of Official Analytical Chemists. Arlington. 19th edition. Arlington, Virginia (USA) 684p.

3. BUREAU, D.P.; HUA, K. 2006. Letter to the editor of aquaculture. Aquaculture. 252:103-105. 
4. CONTRERAS CASTRO, J.H.; CANCHILA ASENCIO, E.R. 2012. Evaluación del rendimiento técnico en cachama blanca Piaractus brachypomus al sustituir morera Morus alba y falso girasol Tithonia diversifolia en el alimento balanceado de ceba. Citecsa. 2(3):1-12.

5. CHO, C.Y.; COWEY, C.B.; WATANABE, T. 1985. Finfish nutrition in Asia: Methodological approaches to research and development. Ottawa, Ont., IDRC. $154 \mathrm{p}$.

6. CRUZ-CASALLAS, P.E.; MEDINA-ROBLES, V.M.; VELASCO-SANTAMARÍA, Y.M. 2011. Fish farming of native species in Colombia: current situation and perspectives. Aquaculture Res. 42(6):823-831.

7. FABREGAT, T.E.H.P.; RODRIGUES, L.A.; TORRES DO NASCIMENTO, T.M.; URBINATI, E.C.; SAKOMURA, N.K.; FERNANDES, J.B.K. 2011. Fontes de fibra na alimentação do pacu: desempenho, composição corporal e morfometria intestinal. Arq. Bras. Med. Vet. Zootec. 63(6):1533-1540.

8. FAO. 2017. Regional review on status and trends in aquaculture development in Latin America and the caribbean - 2015, por Carlos Wurmann G. FAO Fisheries and Aquaculture Circular No. 1135/3. Rome, Italy 36p.

9. FRANCO RODRÍGUEZ, J.E.; BELTRÁN AMARIS, L.M. 2012. Variaciones morfométricas a nivel de las vellosidades en intestino anterior y posterior en cachama blanca (Piaractus brachypomus) con la inclusión de morera (Morus alba) al $15 \%$ en la etapa de ceba. Citecsa. 2(3):50-59.

10. FRANCO RODRÍGUEZ, J.E.; QUINTERO, P.R.N. 2013. Variaciones morfométricas a nivel de intestino anterior y posterior en cachama blanca (Piaractus brachypomus) con base a la inclusión de morera (Morus alba) al $20 \%$ y ensilaje al $20 \%$ en ceba. Citecsa. 4(6):21-30.

11. FRY, J.P.; LOVE, D.C.; MACDONALD, G.K.; WEST, P.C.; ENGSTROM, P.M.; NACHMAN, K.E.; LAWRENCE, R.S. 2016. Environmental health impacts of feeding crops to farmed fish. Envir. Intern. 91:201-214.

12. FURUKAWA, A.; TSUKAHARA, H. 1966. Método de digestão ácida para determinação do óxido crõmo usado como substãncia indica- dora nos estudos de digestibilidade dos alimentos para peixes. Bull. Jap. Soc. Scie. Fisheries. 32(6):502-506.
13. KJELDAHL, J. 1883. A new method for the estimation of nitrogen in organic compounds. Z. Anal. Chem., 22:366.

14. MADR \& IICA. 2012. Agenda nacional de investigación para la pesca y la acuicultura. Bogotá, Colombia, 145p.

15. MAHECHA, L.; ROSALES, M. 2005. Valor nutricional del follaje de botón de oro Tithonia diversifolia (Hemsl.) Gray, en la producción animal en el trópico. Livestock Res. for Rural Devel. 17, Article \#100. 10p.

16. MANRÍQUEZ H., J.A. 1994. La digestibilidad como criterio de evalua- ción de alimentos - Su aplicación en peces y en la conservación del medio ambiente. En: Control de calidad de insumos y dietas acuícolas. Organización de las Naciones Unidas para la Agricultura y la Alimentación - FAO Italia. 7p.

17. NOSE, T. 1966. Recents advances in the study of fish digestión in Japan. Symposium on feeding trout and salmon culture, II, 17 .

18. ORTIZ-GONZÁLEZ, A.R.; MORALES-LUNA, K.A.; VÁSQUEZ-TORRES, W.; GUTIÉRREZ-ESPINOSA, M.C. 2014. Digestibilidad aparente de Tithonia diversifolia, Gliricidia sepium y Cratylia argentea en juveniles de Piaractus brachypomus, Cuvier 1818. Orinoquia. 18(2):214-219.

19. RINCÓN, C.A.; PARDO, B.O.; PARRA, A.J.L.; CERINZA, O.J.; PINZÓN, S.M.; CORREAL, W.A.; ROJAS, B.A. 2007. Establecimiento, manejoyuso dela leguminosa arbustiva forrajera Cratylia argentea cv veranera en el piedemonte llanero. CORPOICA C.I. La Libertad Manual Técnico No.13, Villavicencio Meta. 24p.

20. STEEL, R.G.; TORRIE J.H. 1997. Principles and procedures of statistics: a biometrical approach. Third edition. McGraw-Hill, New York. 672p.

21. SWEETMAN, J.; DIMITROGLOU, A.; DAVIES, S.; TORRECILLAS, S. 2008. Nutrient uptake, gut morphology a key to efficient nutrition. Internatlonal Aquafeed. 1:26-30.

22. VÁSQUEZ-TORRES, W.; PEREIRA FILHO, M.; ARIASCASTELLANOS, J.A. 2002. Estudos para composição de una dieta referência semipurificada para avaliação de exigências nutricionais em juvenis de pirapitinga, Piaractus brachypomus (Cuvier, 1818). Rev. Bras. Zootec. 31:283-292. 
23. VÁSQUEZ, W. 2004. Principios de nutrición aplicada al cultivo de peces. Universidad de los Llanos, Villavicencio. 78p.

24. VÁSQUEZ, W.; YOSSA, M.I.; GUTIÉRREZ-ESPINOSA, M.C. 2013. Digestibilidad aparente de ingredientes de origen vegetal y animal en la cachama. Pesquisa Agrop. Brasileira. 48(8):920-927.

Recibido: Agosto 29 de 2016

Aceptado: Agosto 2 de 2017

Cómo citar:

Puerta Rico, L.F.; García González, J.J.;2, Parra Suescún, J.E.; Pardo Carrasco, S.C. 2017. Coeficientes de digestibilidad aparente de Thitonia diversifolia y Cratylia argentea en cachama blanca y efectos sobre las vellosidades intestinales. Rev. U.D.C.A Act. \& Div. Cient. 20(2): 375-383. 